Supporting Information

\title{
Evolution of Polymer Colloid Structure During Precipitation and Phase Separation
}

Jason X. Liu, ${ }^{\dagger}$ Navid Bizmark,,+ Douglas M. Scott, ${ }^{\ddagger}$ Richard A. Register,,

Mikko P. Haataja, ${ }^{\dagger}$ Sujit S. Datta, ${ }^{\ddagger}$ Craig B. Arnold, ${ }^{\dagger,}$ and Rodney D.

$$
\text { Priestley }{ }^{*,+}, \mathbb{9}
$$

$\dagger$ Department of Mechanical and Aerospace Engineering, Princeton University, Princeton, NJ 08540, USA

$\ddagger$ Department of Chemical and Biological Engineering, Princeton University, Princeton, NJ 08540, USA

IPrinceton Institute for the Science and Technology of Materials, Princeton University, Princeton, NJ 08540, USA

E-mail: rpriest|@princeton.edu

\section{Contents}

1. Materials

2. Experimental methods

3. Electron contrast calculations

4. Free energy calculation methods

5. Continuum simulation methods

6. Movie captions 


\section{Materials}

Tetrahydrofuran (THF) was purchased from Fisher Scientific. DI water was filtered through a $0.2 \mu \mathrm{m}$ filter using a NANOpure Diamond filtration system. Polystyrene (PS) and poly(4-bromostyrene) (PBrS) were purchased from PolymerSource Inc. and used as received. PBrS of molecular weight $M_{n}=143 \times 10^{3} \mathrm{~g} / \mathrm{mol}$ and $M_{w} / M_{n}=1.93$ was synthesized via bulk free-radial polymerization as described below in 2. Experimental methods. PS of $M_{n}=390 \times 10^{3} \mathrm{~g} / \mathrm{mol}$ was obtained from Pressure Chemical Co. Benzoyl peroxide initiator was purchased from Sigma Aldrich. Methanol was purchased from Sigma Aldrich. Aluminum oxide powder was purchased from Sigma Aldrich.

The polymer blends employed in Figure 1 of the main text (and Supporting Figure S1) are $\left(N_{P B r S}\right.$ is calculated with a styrene monomer as the reference volume):

Figure 1a: PS: $M_{n}=0.95 \mathrm{k} ; N_{P S}=9 ; M_{w} / M_{n}=1.2 ; \operatorname{PBrS}: M_{n}=2.5 \mathrm{k} ; N_{P B r S}=11 ; M_{w} / M_{n}=1.28$

Figure 1b: PS: $M_{n}=3.2 \mathrm{k} ; N_{P S}=31 ; M_{w} / M_{n}=1.11 ; \mathrm{PBrS}: M_{n}=5.6 \mathrm{k} ; N_{P B r S}=26 ; M_{w} / M_{n}=1.2$

Figure 1c: PS: $M_{n}=16 \mathrm{k} ; N_{P S}=154 ; M_{w} / M_{n}=1.03 ;$ PBrS: $M_{n}=24 \mathrm{k} ; N_{P B r S}=110 ; M_{w} / M_{n}=1.4$

Figure 1d: PS: $M_{n}=390 \mathrm{k} ; N_{P S}=3750 ; M_{w} / M_{n}=1.10 ;$ PBrS: $M_{n}=143 \mathrm{k} ; N_{P B r S}=657 ; M_{w} / M_{n}=1.93$

The asymmetric blends used in Figure $\mathbf{6}$ of the main text are:

Figure 6a: PS: $M_{n}=0.95 \mathrm{k} ; N_{P S}=9 ; M_{w} / M_{n}=1.2 ; \operatorname{PBrS:} M_{n}=24 \mathrm{k} ; N_{P B r S}=110 ; M_{w} / M_{n}=1.4$

Figure 6b: PS: $M_{n}=3.2 \mathrm{k} ; N_{P S}=31 ; M_{w} / M_{n}=1.11 ; \operatorname{PBrS:} M_{n}=5.6 \mathrm{k} ; N_{P B r S}=26 ; M_{w} / M_{n}=1.2$

Figure 6c: PS: $M_{n}=6 \mathrm{k} ; N_{P S}=58 ; M_{w} / M_{n}=1.10 ; \operatorname{PBrS}: M_{n}=5.6 \mathrm{k} ; N_{P B r S}=26 ; M_{w} / M_{n}=1.2$

\section{Experimental methods}

To form colloidal suspensions, we first prepared $0.5 \mathrm{mg} / \mathrm{mL}$ solutions of PS and $\mathrm{PBrS}$ separately in THF. Solutions were filtered through a $0.2 \mu \mathrm{m}$ PTFE filter and $1 \mathrm{~mL}$ of each solution was loaded into dialysis tubing $(2 \mathrm{~mL}$ total volume, $0.5 \mathrm{mg} / \mathrm{mL}$ solids content, 1:1 $\mathrm{PS} / \mathrm{PBrS}$ mass ratio). To precipitate the colloids, the dialysis tubing was placed into a $2 \mathrm{~L}$ beaker of water with light stirring for at least 6 hours. Regenerated cellulose dialysis membranes with molecular weight cutoff between $6-8 \mathrm{kDa}(\sim 2 \mathrm{~nm}$ pore size) were obtained from Spectrum Labs. No diffusion of PS or PBrS into the aqueous phase was expected due to its low solubility in the aqueous phase as well as due to precipitation of particles which were larger than the dialysis membrane pore size. ${ }^{1}$ After precipitation, $5 \mu \mathrm{L}$ of the suspensions, now in water, were drop cast onto carbon film TEM grids. TEM imaging was performed on an FEI Talos operating at $200 \mathrm{kV}$. Additional examples of particle morphologies are shown in Supporting Figure S1 below.

To measure particle size distributions, the precipitated suspensions were diluted to 0.05 
$\mathrm{mg} / \mathrm{mL}$ for dynamic light scattering (DLS). Measurements were performed on an Anton Paar Litesizer 500 with a laser wavelength of $658 \mathrm{~nm}$, from a single-frequency laser diode operating at $40 \mathrm{~mW}$. The average hydrodynamic diameter and standard deviation reported in Supporting Figure S1 is obtained from 5 measurements.

PBrS of number-averaged molecular weight $M_{n}=143 \times 10^{3} \mathrm{~g} / \mathrm{mol}$ and $M_{w} / M_{n}=1.93$ was synthesized via bulk free-radial polymerization of 4 -bromostyrene at $90{ }^{\circ} \mathrm{C}$ under nitrogen with benzoyl peroxide as the initiator. Prior to polymerization, the monomer was passed through a packed aluminum oxide column to remove the inhibitor. After polymerization, the polymer was dissolved in THF and precipitated in methanol three times to remove excess monomer. Molecular weight and polydispersity were measured by gel permeation chromatography in THF relative to polystyrene standards.

The surface energies of PS and PBrS were determined by dynamic measurements of water contact angles on their corresponding films. The polymer films were prepared by spin-coating 2 wt.\% solutions of PS or PBrS in toluene at $1000 \mathrm{rpm}$ for $60 \mathrm{~s}$ onto silicon wafers. The polymer film thickness was expected to be $500 \mathrm{~nm} .{ }^{2}$ Prior to water contact angle measurements, the polymer films were annealed at $120{ }^{\circ} \mathrm{C}$ overnight in a vacuum oven. A $5 \mathrm{~mL}$ drop of mili-Q water was placed on the polymer-coated silicon wafers and the contact angle was measured for 10 min using a drop shape analyzer (DSA30 by Krüss). With three replicates, the water contact angle measured on PS and PBrS films was $92 \pm 1.5^{\circ}$ and $93 \pm 3^{\circ}$, respectively, with no change over time. Therefore, PS and PBrS were expected to have similar interfacial energies with water and the equilibrium colloid morphology is the Janus structure. ${ }^{3}$

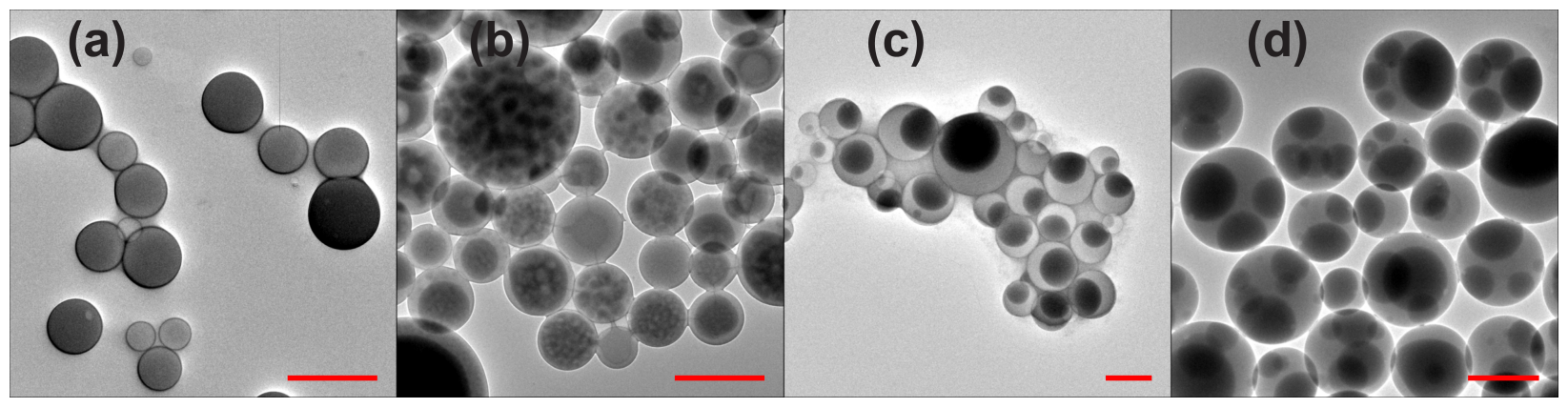

Figure S1: Additional TEM images of colloid morphologies. Experimental degrees of polymerization are the same as those of Figure $\mathbf{1}$ in the main text. They are: (a) $N_{P S}=$ $9, N_{P B r S}=11$, (b) $N_{P S}=31, N_{P B r S}=26$, (c) $N_{P S}=154, N_{P B r S}=110$, and (d) $N_{P S}=$ $3750, N_{P B r S}=657$. The mean hydrodynamic diameter and standard deviation as measured by DLS are: (a) $505 \pm 38 \mathrm{~nm}$, (b) $582 \pm 44 \mathrm{~nm}$, (c) $821 \pm 251 \mathrm{~nm}$, and (d) $742 \pm 95 \mathrm{~nm}$. Dark regions correspond to PBrS and light regions to PS. Scale bars are $1 \mu \mathrm{m}$. 


\section{Electron contrast calculations}

In amorphous materials, contrast in the TEM is generated by differences in incoherent elastic scattering of electrons by the material. Contrast is defined as a percentage, and it is the difference in measured intensity between adjacent areas divided by the intensity at one of the areas: ${ }^{4}$

$$
C=\frac{I_{2}-I_{1}}{I_{1}}=\frac{\Delta I}{I_{1}}
$$

Where $I_{1}$ and $I_{2}$ are the beam intensities after passing through different areas of a sample. Assuming a standard exponential attenuation of the beam intensity by incoherent elastic scattering, we have:

$$
\begin{aligned}
& I_{1}=I_{0,1} \cdot \exp \left(-n_{1} \cdot t_{1} \cdot \sigma_{\text {nucl }, 1}\right) \\
& I_{2}=I_{0,2} \cdot \exp \left(-n_{2} \cdot t_{2} \cdot \sigma_{\text {nucl }, 2}\right)
\end{aligned}
$$

Where $I_{0,1}=I_{0,2}=I_{0}$ is the initial beam intensity, $n_{1}$ and $n_{2}$ are the number density of scattering centers, $t_{1}$ and $t_{2}$ are the thicknesses of the two regions, and $\sigma_{\text {nucl,1 }}$ and $\sigma_{\text {nucl, } 2}$ are the scattering cross sections of the individual scattering centers. The number density of scattering centers can be calculated by using $n=\rho N_{a} / M$ where $\rho$ is the mass density, $N_{a}$ is Avogadro's number, and $M$ is the molecular weight. For these calculations, we use the molecular weight of the polymer's monomer unit which is $104.15 \mathrm{~g} / \mathrm{mol}$ for PS and 183.05 $\mathrm{g} / \mathrm{mol}$ for PBrS. The density of PS is taken as $1.05 \mathrm{~g} / \mathrm{cc}$ and that of PBrS as $1.67 \mathrm{~g} / \mathrm{cc}^{5}$ Thus, by considering a single polymer monomer unit as an individual scattering center, this yields $n_{\mathrm{PS}}=6.07 \times 10^{27} \mathrm{~m}^{-3}$ for PS and $n_{\mathrm{PBrS}}=5.49 \times 10^{27} \mathrm{~m}^{-3}$ for PBrS.

The total elastic scattering cross section is given by: 6,7

$$
\sigma_{\mathrm{el}}=\frac{h^{2} Z^{4 / 3}}{\pi m_{0}^{2} v^{2}\left(1+\left(\frac{\alpha}{\theta_{0}}\right)^{2}\right)}
$$

where the characteristic screening angle in radians is $\theta_{0}=\lambda / 2 \pi R . \mathrm{R}$ is the $Z$-equivalent Bohr radius, $R=a_{0} Z^{-1 / 3}$. Here, $a_{0}$ is the Bohr radius of hydrogen, $53 \mathrm{pm}$, and $Z$ is the atomic number. The electron wavelength, $\lambda$, is $2.51 \mathrm{pm}$ for a $200 \mathrm{keV}$ electron beam. The scattering cross sections of the monomer units can be calculated as a sum of the monomer's constituent's atomic cross sections. The monomer of $\mathrm{PS}$ is $\left(\mathrm{C}_{8} \mathrm{H}_{8}\right)_{n}$ and of $\mathrm{PBrS}$ is $\left(\mathrm{C}_{8} \mathrm{H}_{7} \mathrm{Br}\right)_{n}$. This yields:

$$
\begin{gathered}
\sigma_{\mathrm{PS}}=8 \sigma_{\mathrm{C}}+8 \sigma_{\mathrm{H}}=\left(8 \cdot 3.74 \times 10^{-23}+8 \cdot 2.69 \times 10^{-24}\right) \mathrm{m}^{2}=3.2 \times 10^{-22} \mathrm{~m}^{2} \\
\sigma_{\mathrm{PBrS}}=8 \sigma_{\mathrm{C}}+7 \sigma_{\mathrm{H}}+1 \sigma_{\mathrm{Br}}=\left(8 \cdot 3.74 \times 10^{-23}+7 \cdot 2.69 \times 10^{-24}+1 \cdot 4.3 \times 10^{-22}\right) \mathrm{m}^{2}=7.5 \times 10^{-22} \mathrm{~m}^{2}
\end{gathered}
$$


In Supporting Figure S2, we plot the electron beam's relative intensity as well as contrast, $C=\Delta I / I$, as a function of thickness through a PS vs. PBrS layer. Based on these calculations, we demonstrate that PBrS can generate ample contrast relative to PS in the TEM.

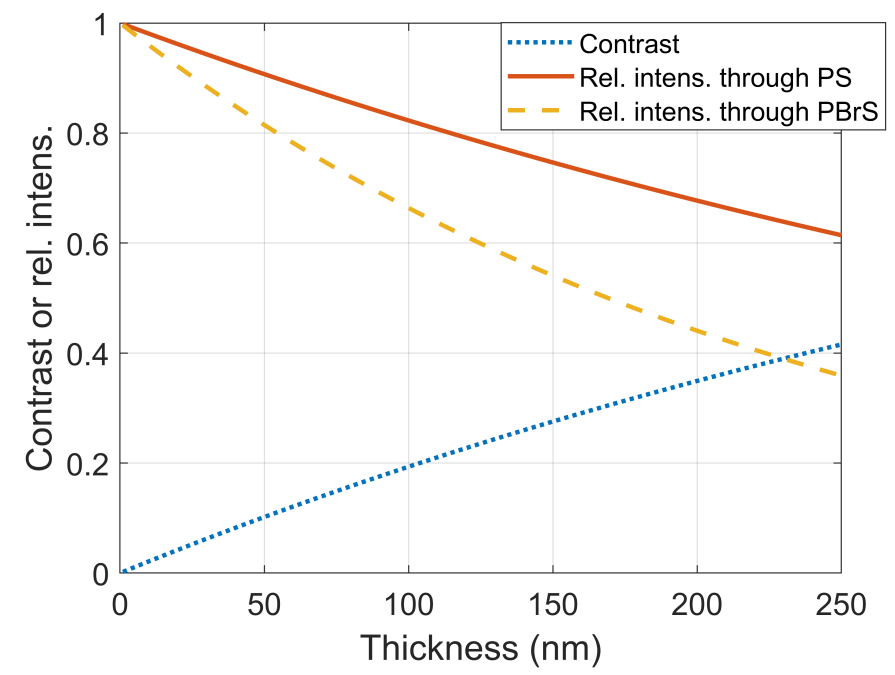

Figure S2: Electron beam relative intensity through varying thicknesses of PS and PBrS, solid and dashed lines. Thickness dependence of image contrast between sections of PS and PBrS, dotted line. Values calculated for a $200 \mathrm{keV}$ electron beam.

\section{Free energy calculation methods}

We performed free-energy calculations using the Flory-Huggins solution theory which describes the free energy of mixing for an $N$-component mixture of polymers and solvents. ${ }^{3,8,9}$

$$
f=c_{0} R T\left[\sum_{i=1}^{N} \frac{\phi_{i} \log \phi_{i}}{N_{i}}+\frac{1}{2} \sum_{i, j=1}^{N} \chi_{i j} \phi_{i} \phi_{j}\right]
$$

where $f$ is the free energy density of mixing, $c_{0}$ is the molar concentration of species, $R$ is the gas constant, $T$ is the absolute temperature, $\phi_{i}$ is the component- $i$ volume fraction, $N_{i}$ is the component- $i$ degree of polymerization, and $\chi_{i j}$ is the interaction parameter between components $i$ and $j$.

We employed this theory with three components: polymers $A$ and $B$, and $S$, which is an effective solvent that models the solvent and antisolvent together. The interaction parameter between polymers $A$ and $B$ was fixed at $\chi_{A B}=0.15$, and the interaction parameter between the effective solvent and both polymers was equal: $\chi_{A S}=\chi_{B S}=\chi_{P S}$. The initial solution concentration of both polymers was $\phi_{A 0}=\phi_{B 0}=0.015$. Ternary phase diagrams were calculated for different values of $\chi_{P S}$ to depict the phase diagram at varying extents of solvent mixing. 
We calculated ternary phase diagrams by applying the convex hull algorithm ${ }^{10}$ to the free energy surface. This is the generalization of the common tangent construction to multiple components. One-, two-, and three-phase coexistence regions were identified by a comparison of edge lengths of the facets created by triangulating the convex hull construction. ${ }^{3}$

In Supporting Figure S3, we additionally depict the evolving phase diagram for the $N_{A, B}=15$ case, in which a homogeneous colloid persists, as described in the main text. During solvent exchange, the starting composition, marked by the cyan circle, undergoes liquid-liquid phase separation into a polymer-rich and polymer-poor phase. The limit of solubility is reached at $\chi_{P S}=0.95$, and the composition of the initial precipitate is marked with the purple triangle. As $\chi_{P S}$ increases, the polymer volume fraction of the condensate rises. However, the vitrification volume fraction, at the black dashed line, is reached prior to polymer-polymer phase separation, at the yellow square. Thus, the colloid is kinetically arrested in a homogeneous state.

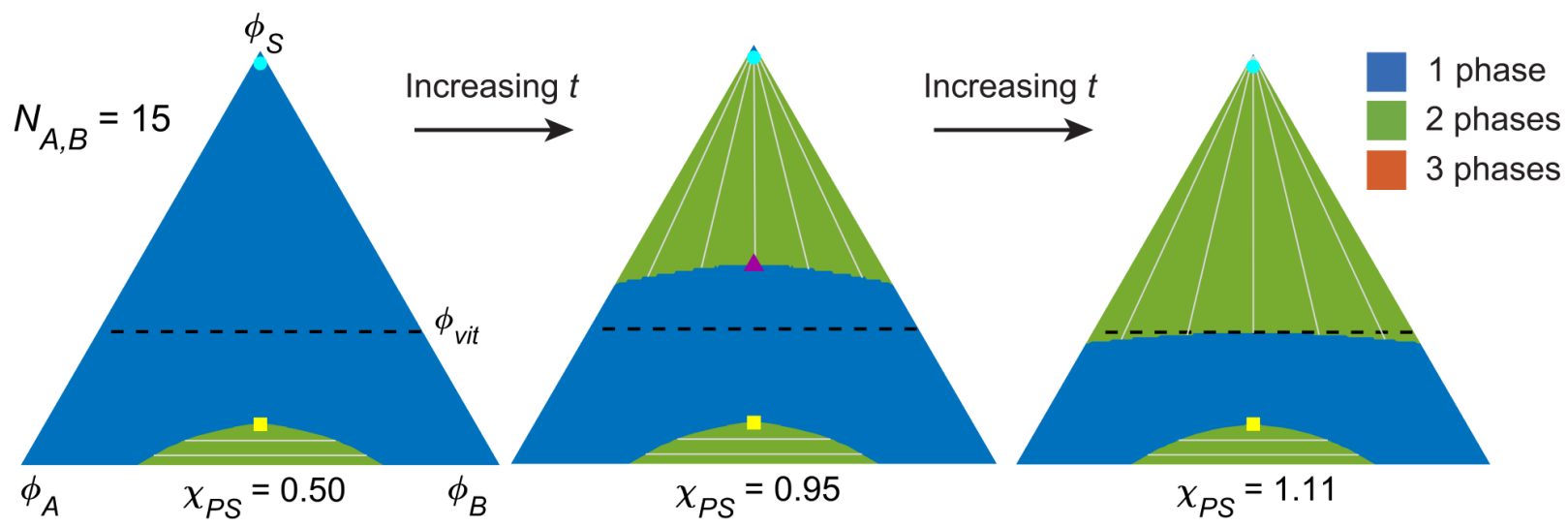

Figure S3: Temporal evolution of the ternary phase diagram for $N_{A, B}=15$. The initial solution composition is marked by the cyan circle. The composition of the initial precipitate is marked by the purple triangle. The critical volume fraction required for polymer-polymer phase separation is marked by the yellow square. Since the dashed black vitrification line is reached prior to the critical volume fraction, only a homogeneous condensate results.

In Supporting Figure S4, we depict $\phi_{c}, \phi_{P 0}$, and $\phi_{v i t}$ as a function of $N_{A, B}$ for the specific case of $\chi_{A B}=0.15$ to illustrate how the phase diagram of Figure 3 was generated. $\phi_{c}\left(N_{A, B}\right)$ is generated by considering the Hessian stability matrix, ${ }^{8,11} H$, of the Flory-Huggins free energy, $f$.

$$
H=\left[\begin{array}{ll}
f_{A A} & f_{A B} \\
f_{B A} & f_{B B}
\end{array}\right]=\left[\begin{array}{cc}
\frac{\partial f}{\partial \phi_{A}^{2}} & \frac{\partial f}{\partial \phi_{A} \partial \phi_{B}} \\
\frac{\partial f}{\partial \phi_{B} \partial \phi_{A}} & \frac{\partial f}{\partial \phi_{B}^{2}}
\end{array}\right]
$$


To locate $\phi_{c}$, at the apex of the spinodal curve, we set the determinant of $H$ equal to zero:

$$
|H|=\left(\frac{1}{\phi_{A} N_{A}}+\frac{1}{\phi_{S} N_{S}}-\chi_{A S}\right) \cdot\left(\frac{1}{\phi_{B} N_{B}}+\frac{1}{\phi_{S} N_{S}}-\chi_{B S}\right)-\left(\frac{1}{\phi_{S} N_{S}}+\chi_{A B}-\chi_{A S}-\chi_{B S}\right)^{2}=0
$$

Solving the above equation at different values of $N_{A, B}$ yields $\phi_{c, A}$ and $\phi_{c, B}$, the values at which the critical point is located $\left(\phi_{c}=\phi_{c, A}+\phi_{c, B}\right.$ where $\left.\phi_{c, A}=\phi_{c, B}\right)$.

We calculate $\phi_{P 0}$ by using the Flory-Huggins free energy, $f$, by first setting $\phi_{A}=\phi_{B}=$ $\frac{\phi_{P}}{2}=\frac{1-\phi_{S}}{2}$, where $\phi_{P}$ is the polymer volume fraction. For the free energy $f$, this yields:

$$
f=\frac{\left(1-\phi_{S}\right) \ln \left(\left(1-\phi_{S}\right) / 2\right)}{N_{A, B}}+\phi_{S} \ln \phi_{S}+\left(\frac{1-\phi_{S}}{2}\right)^{2} \chi_{A B}+\left(1-\phi_{S}\right) \phi_{S} \chi_{P S}
$$

Then, at various values of $N_{A, B}$, we use the Maxwell construction to determine at what $\chi_{P S}$ value the starting composition, $\phi_{0, A}+\phi_{0, B}=0.03$, intersects the binodal (i.e. when precipitation first occurs). The polymer-rich composition at this value of $\chi_{P S}$ yields $\phi_{P 0}$. Then, to determine the different pathways of particle formation, we compare $\phi_{c}, \phi_{P 0}$, and $\phi_{v i t}$. Please refer to the caption of Supporting Figure S4 for details.

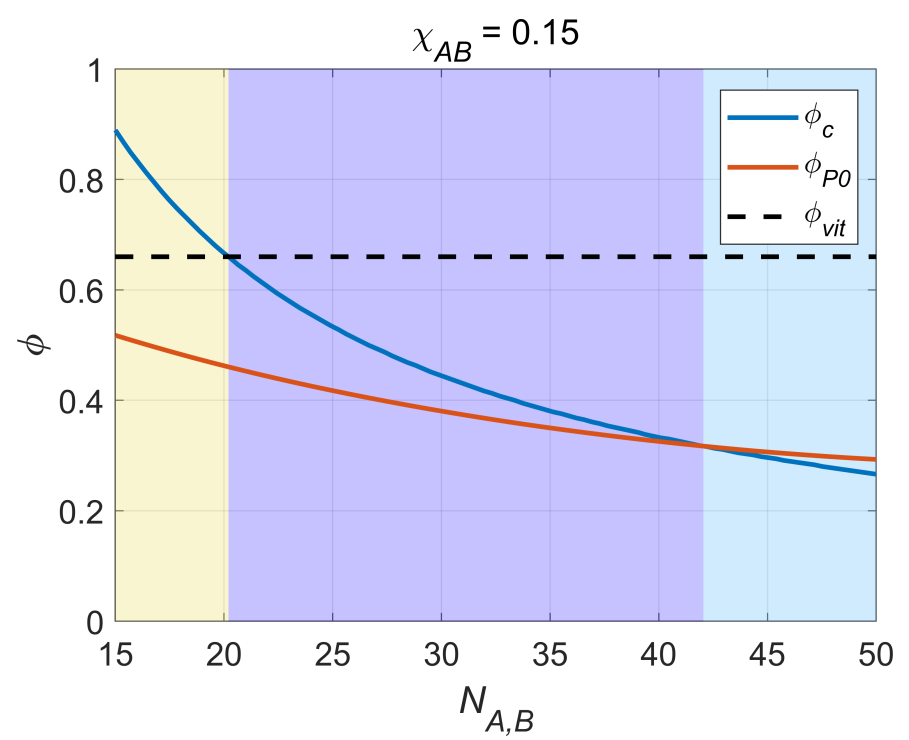

Figure S4: Depiction of the critical volume fraction for $A-B$ phase separation $\phi_{c}$, the precipitate's initial volume fraction $\phi_{P 0}$, and the vitrification volume fraction, $\phi_{v i t}=0.66$ as a function of molecular weight for $\chi_{A B}=0.15$. Within the blue region, diffusion and vitrification compete to determine the final structure, with $\phi_{c}<\phi_{P 0}<\phi_{v i t}$. The purple region indicates colloids that can spinodally decompose into a bicontinuous structure, $\phi_{P 0}<$ $\phi_{c}<\phi_{v i t}$, and the yellow region indicates colloids that vitrify in the homogeneous state, $\phi_{P 0}<\phi_{v i t}<\phi_{c}$. The phase diagram in Figure 3 of the main text is generated with such a comparison of the different volume fractions at different values of $\chi_{A B}$ and $N_{A, B}$. 


\section{Continuum simulation methods}

We further modified the Flory-Huggins free energy with a gradient energy term penalizing the formation of an interface between two dissimilar materials. ${ }^{12}$

$$
f=c_{0} R T\left[\sum_{i=1}^{N} \frac{\phi_{i} \log \phi_{i}}{N_{i}}+\frac{1}{2} \sum_{i, j=1}^{N} \chi_{i j} \phi_{i} \phi_{j}-\frac{\lambda^{2}}{2} \sum_{i, j=1}^{N} \chi_{i j} \nabla \phi_{i} \nabla \phi_{j}\right]
$$

where $\lambda$ is the characteristic width of an interface. $\lambda^{2} \chi_{i j}$ describes the energetic penalty incurred in the formation of an interface.

To model the kinetics of colloid growth and phase separation, we employed the generalized diffusion equation to describe mass transport driven by the above free energy. This transport equation takes into account species' chemical potential gradients and is given by

$$
\frac{\partial \phi_{i}}{\partial t}=\nabla \cdot\left[D(\phi) \cdot \phi_{i} \sum_{j}\left(\delta_{i j}-\phi_{j}\right) \nabla \mu_{j}\right]
$$

where $D(\phi)=D_{S}^{\phi_{S}} \cdot D_{P}^{\phi_{P}}$ is a concentration-dependent diffusion coefficient which is modeled as a weighted geometric mean of the solvent and polymer diffusivities where $D_{S}$ is maintained at 1024. $\delta_{i j}$ is the Kronecker delta function, and $\mu_{j}$ is the chemical potential. Here, $\phi_{i} \sum_{j}\left(\delta_{i j}-\phi_{j}\right)$ represents a mobility which takes into account compositional fluxes to ensure incompressibility, ${ }^{13}$ namely that $\phi_{A}+\phi_{B}+\phi_{S}=1$. The chemical potential is obtained from the free energy density, $f$, via

$$
\mu_{i}=\frac{\delta f}{\delta \phi_{i}}=\frac{1}{N_{i}}+\frac{\log \phi_{i}}{N_{i}}+\sum_{j=1}^{N} \chi_{i j}\left(1+\lambda^{2} \nabla^{2}\right) \phi_{j}
$$

To defeat the stiffness of the Cahn-Hilliard equation, we employed a stabilized, implicitexplicit Fourier pseudo-spectral numerical scheme to calculate the time evolution of the system. ${ }^{14-16}$ In particular, first we discretized Equation 12 with a first-order forward difference in time. Then we separated the right hand side into linear, $L_{i}$, and nonlinear $M_{i}$, terms:

$$
\frac{\phi_{i}^{n+1}-\phi_{i}^{n}}{\Delta t}=M_{i}\left(\phi_{i}^{n}\right)+L_{i}\left(\phi_{i}^{n+1}\right)
$$

Following the technique used by others, ${ }^{3,15,16}$ the following stabilizing linear term was employed,

$$
L_{i}\left(\phi_{i}\right)=-A \bar{D} \lambda^{2} \nabla^{4} \phi_{i}
$$

Where $A=0.5\left(\chi_{A S}+\chi_{B S}+\chi_{A B}\right) / 3$ and $\bar{D}$ is the composition-dependent diffusion coefficient 
averaged across the entire simulation domain. We employed the fast Fourier transform to evaluate the discretized Equation 14 numerically

$$
\hat{\phi}_{i}^{n+1}=\frac{\hat{\phi}_{i}^{n}+\hat{M}_{i}\left(\phi_{i}^{n}\right) \Delta t}{1+A \lambda^{2} k^{4} \bar{D} \Delta t}
$$

where $k$ is the magnitude of the wavevector and ${ }^{\wedge}$ indicates the Fourier transform of a quantity.

All simulations were performed in a domain of size $L=200$, with $512 \times 512$ grid points, and with characteristic lengthscale $\lambda=0.5$. Time was measured in units of $\tau=\lambda^{2} / D_{S}$ and a time step of $d t=1 \tau$ was taken at every iteration. Simulations were initialized at $\chi_{0}$, the value of the $\chi_{P S}$ interaction parameter at which the initial background concentration, $\phi_{0, A}=\phi_{0, B}=0.015$, has reached the limit of solubility for a given blend degree of polymerization. This corresponds to $\chi_{0}=0.95,0.83$, and 0.72 for $N_{A, B}=15,25$, and 50, respectively. The simulation was initialized at this point with a homogeneous, supercritical nucleus of radius $r=5$, whose polymer volume fraction was that of the polymer-rich phase precipitated at $\chi_{0}: \phi_{P 0}$. These values were calculated from the ternary phase diagram and are $\phi_{P 0}=$ $0.51,0.41$, and 0.33 for $N_{A, B}=15,25$, and 50, respectively. The initial nucleus' polymer volume fraction is evenly distributed between $A$ and $B: \phi_{0, A, n u c l e u s}=\phi_{0, B, \text { nucleus }}=\phi_{P 0} / 2$. The largest degree of polymerization we simulated was $N_{A, B}=50$. This was to avoid numerical difficulties associated with the extremely low equilibrium dilute phase concentration. At high $N_{A, B}$, prohibitively small time steps must be taken to ensure that the simulation composition does not attain a negative value. Temporally and spatially uncorrelated Gaussian random noise with zero mean and amplitude $0.03 \times \phi_{i}\left(1-\phi_{i}\right)$ was used to emulate thermal fluctuations. As discussed in the main text, we modeled the solvent exchange process by increasing the polymer-solvent interaction parameter, $\chi_{A S}=\chi_{B S}=\chi_{P S}$, linearly with time with slope $c: \chi_{P S}(t)=\chi_{0}+c t$. We used the same slope, $c=6.27 \times 10^{-8} / \tau$ in all simulations. $\chi_{A B}$ was maintained at 0.15 for all simulations. Simulations were run until vitrification was reached at $\phi_{v i t}=0.66$, typically around $t=4 \times 10^{6} \tau$. All codes were written in MATLAB. 


\section{Movie captions}

"Fluxes D_AB=64, N_AB=50.mp4": Fluxes and composition of polymer $A$ with $N_{A, B}=50$. Polymer diffusivity is $D_{A, B}=64$ and a patchy colloid forms.

"Fluxes D_AB $=\mathbf{5 1 2}, \mathbf{N} \_\mathbf{A B}=\mathbf{5 0 . m p 4 " : ~ F l u x e s ~ a n d ~ c o m p o s i t i o n ~ o f ~ p o l y m e r ~} A$ with $N_{A, B}=50$. Polymer diffusivity is $D_{A, B}=512$ and a Janus colloid forms.

"N=15 homogeneous.mp4": Morphology evolution for $N_{A, B}=15$ alongside the ternary phase diagram. Red corresponds to polymer $A$, green to polymer $B$, blue to the solvent $S$, and gray to a homogeneous mixture of $A$ and $B$. A homogeneous colloid forms.

"N=25 spinodal.mp4": Same, for $N_{A, B}=25$, where a bicontinuous colloid forms.

"N=50 Janus.mp4": Same, for $N_{A, B}=50$ and $D_{A, B}=512$, where a Janus colloid forms.

"N=50 patchy.mp4": Same, for $N_{A, B}=50$ and $D_{A, B}=64$, where a patchy colloid forms. 


\section{References}

(1) Liu, Y.; Kathan, K.; Saad, W.; Prud'homme, R. K. Ostwald ripening of $\beta$-carotene nanoparticles. Physical Review Letters 2007, 98, 8-11.

(2) Hall, D. B.; Underhill, P.; Torkelson, J. M. Spin Coating PS. Polymer Engeneering and Science 1998, 38, 2039-2045.

(3) Mao, S.; Kuldinow, D.; Haataja, M. P.; Košmrlj, A. Phase behavior and morphology of multicomponent liquid mixtures. Soft Matter 2019, 15, 1297-1311.

(4) Williams, Transmission Electron Microscopy; 2009.

(5) Strobl, G. R.; Bendler, J. T.; Kambour, R. P.; Shultz, A. R. Thermally Reversible Phase Separation in Polystyrene/Poly(styrene-co-4-bromostyrene) Blends. Macromolecules 1986, 19, 2683-2689.

(6) Reimer, L. Transmission Electron Microscopy; 2008.

(7) Ianiro, A.; Wu, H.; van Rijt, M. M.; Vena, M. P.; Keizer, A. D.; Esteves, A. C. C.; Tuinier, R.; Friedrich, H.; Sommerdijk, N. A.; Patterson, J. P. Liquid-liquid phase separation during amphiphilic self-assembly. Nature Chemistry 2019, 11, 320-328.

(8) Huang, C.; de la Cruz, M. O.; Swift, B. W. Phase Separation of Ternary Mixtures: Symmetric Polymer Blends. Macromolecules 1995, 28, 7996-8005.

(9) Colby, R. H.; Rubinstein, M. Polymer Physics; 2003.

(10) Barber, C. B.; Dobkin, D. P.; Huhdanpaa, H. The Quickhull Algorithm for Convex Hulls. ACM Transactions on Mathematical Software 1996, 22, 469-483.

(11) Solokhin, M. A.; Solokhin, A. V.; Timofeev, V. S. Phase-equilibrium stability criterion in terms of the eigenvalues of the hessian matrix of the Gibbs potential. Teoreticheskie Osnovy Khimicheskoi Tekhnologii 2002, 36, 487-491.

(12) Cahn, J. W. Free energy of a nonuniform system. II. Thermodynamic basis. The Journal of Chemical Physics 1959, 30, 1121-1124.

(13) Kramer, E. J.; Green, P.; Palmstrøm, C. J. Interdiffusion and marker movements in concentrated polymer-polymer diffusion couples. Polymer 1984, 25, 473-480.

(14) Trefethen, L. Spectral methods in Matlab; 2000. 
(15) Zhu, J.; Chen, L. Q.; Shen, J.; Tikare, V. Coarsening kinetics from a variable-mobility cahn-hilliard equation: Application of a semi-implicit fourier spectral method. Physical Review E - Statistical Physics, Plasmas, Fluids, and Related Interdisciplinary Topics 1999, 60, 3564-3572.

(16) Cogswell, D. a. Thesis: A phase-field study of ternary multiphase microstructures. 2010, 179 . 